Ann. Génét. Sél. anim., I97I, 3 (3), 235-243.

\title{
LA MALATE DÉSHYDROGÉNASE MITOCHONDRIALE : VARIANTS ÉLECTROPHORÉTIQUES DE NATURE HÉRÉDITAIRE DANS L'ESPËCE BOVINE $\left({ }^{*}\right)$
}

\author{
M. ANSAY, R. HANSET et J. ESSER-COULON \\ Chaive de Génétique, Faculté de Médecine vétérinaive (Cuveghem-Bruxelles), \\ Université de Liège, \\ Rue des Vétérinaires 45, Io7o, Bruxelles
}

\begin{abstract}
RÉSUMÉ
Un polymorphisme de l'isozyme mitochondrial de la malate déshydrogénase (E.C.1.1.1.37) est décrit dans l'espèce bovine. Le svstème se compose de deux allèles codominants. Les trois phénotypes sont symbolisés par Mor-1-BB, Mor-1-AB, Mor-1-AA. Dans les sept races bovines étudiées, la fréquence de l'allèle Mor-1-A, correspondant à la forme la moins commune de la malate déshydrogénase, s'est située entre 0 et $8,6 \%$.
\end{abstract}

L'emploi conjugué de deux techniques : Io l'électrophorèse de zone, dans ses variantes multiples (SmithiEs, I955, I959; KOHN, I957; WIEME, I965); $2^{\circ}$ les méthodes de colorations histochimiques (HUNTER et MARKERT, I957) a révélé que la plupart des enzymes tissulaires existaient sous plusieurs formes. Le mot isozyme (ou isoenzyme) employé tout d'abord par MARKERT et MOLLER (I959) est un terme opérationnel employé pour décrire les différentes molécules trouvées dans le même organisme et catalysant la même réaction (SHAw, I964).

La Malate déshydrogénase (L.-Malate : NAD oxydoréductase E.C.I.I.I.37) (Fior KIN et STOTz, I964) catalyse, dans le cycle citrique, la réaction suivante :

$$
\text { L.-Malate }+\mathrm{NAD} \rightleftharpoons \text { oxaloacétate }+\mathrm{NADH}
$$

Elle est présente dans la cellule sous deux formes, caractérisées d'abord par leur localisation : cytoplasmique et mitochondriale.

(*) Travail exécuté sous les auspices de l'Institut pour l'Encouragement de la Recherche Scientifique dans l'Industrie et l'Agriculture (I.R.S.I.A.). 
L'individualité de chacun de ces deux isoenzymes ressort davantage encore quand d'autres critères de caractérisation sont employés : mobilité électropborétique, chromatographie, inhibition par le substrat (THORNe et al., I963; THORNE, I960; KIтTo, I969).

L'objet de ce travail est de montrer l'existence, chez les bovidés, de variants électrophorétiques de la malate déshydrogénase mitochondriale, de préciser leur déterminisme héréditaire et d'estimer leur fréquence dans les races bovines exploitées en Belgique.

\section{MATÉRIEL ET MÉTHODES}

\section{Extraits musculaives}

Effectués dans diverses régions de la musculature squelettique, les prélèvements musculaires sont homogénéisés à l'aide d'un appareil Ultra-Turrax, dans un tampon $\mathrm{KH}_{2} \mathrm{PO}_{4}-\mathrm{Na}_{2} \mathrm{HPO}_{4}$ $0,1 \mathrm{M} \mathrm{pH} \mathrm{7,25.} \mathrm{Le} \mathrm{rapport} \mathrm{muscle/tampon} \mathrm{est} \mathrm{de} 1 / 4$.

Le surnageant récupéré après une centrifugation à $100000 \mathrm{~g}$ pendant 20 minutes, est soumis à l'électrophorèse.

\section{Extraits leucocytaives}

Le sang est recueilli sur EDTAdiNA pH 7.0, 0,155 M $(5 \mathrm{ml} / 50 \mathrm{ml}$ de sang). Après hémolyse des globules rouges par $\mathrm{NH}_{4} \mathrm{Cl} 0,155 \mathrm{M}(70 \mathrm{ml} / 20 \mathrm{ml}$ de sang), les globules blancs sont séparés par centrifugation à $2000 \mathrm{~g}$. Le culot, additionné de $0,3 \mathrm{ml}$ de tampon Phosphate $0,1 \mathrm{M}, \mathrm{pH} 7,25$, est congelé, décongelé plusieurs fois, homogénéisé à l'Ultra-Turrax puis centrifugé.

\section{Électrophorèses}

- L'électrophorèse sur acétate de cellulose est pratiquéc à l'aide de bandes de celloge. $(5,7 \times 14 \mathrm{~cm}$; épaisseur 200 microns $)$. On dépose $0,5 \mu l$ par $0,5 \mathrm{~cm}$ de l'échantillon à analyser.

L'électrophorèse est conduite sous une tension de $240 \mathrm{~V}$ (mesurée à la sortie de l'appareil)

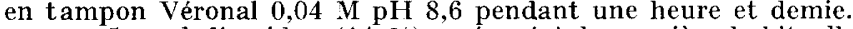

- Le gel d'amidon $(14 \%$ ), préparé à la manière habituelle (SMithies, 1955) est coulé en plaques minces $(1,5 \mathrm{~mm}$ d'épaisseur; $9 \times 12 \mathrm{~cm}$ ) suivant une technique déjà décrite (ANSAY, 1962).

Une plaque de refroidissement au sein de laquelle circule de l'eau à $0{ }^{\circ} \mathrm{C}$ prévient l'élévation de température.

- L'électrophorèse en gel d'acrylamide $(7,5 \%)$ est pratiquée de la même façon. La polymérisation s'effectue entre deux plaques de verre suivant un procédé décrit par URIEL (1966) mais sans adjonction de gélose. Les plaques $(9 \times 12 \mathrm{~cm})$ sont découpées puis rincées et imbibées de tampon (Wieme, 1965).

Pour les gels d'amidon et d'acrylamide, le tampon employé est celui de Srows et al. (1969); les insertions sont réalisées au milieu de la plaque.

\section{Révélatian}

1) La première méthode de révélation du zymogramme, méthode habituelle et dite "positive ", basée sur la réduction de sels de tétrazolium par le nicotinamide adénine dinucléotide réduit $\left(\mathrm{NADH}_{2}\right)$ est effectuée à l'aide d'un gel à base de gélose et contenant tous les réactifs. Sur ce gel, on étale la bande d'acétate de cellulose. Les plaques d'amidon ou d'acrylamide sont, au contraire, recouvertes de ce gel.

La composition du mélange réactionnel $(10 \mathrm{ml})$, exprimée en concentration finale est :

- gélose : $1 \%$

-- tampon Tris-HCl pH 7,2 : $70 \mathrm{mM}$

- acide D.L. malique (Merck) $0,1 \mathrm{M}$, neutralisé à $\mathrm{pH} 7,2$ avec $\mathrm{Na}_{2} \mathrm{CO}_{3}$

-- Nicotinamide adénine nucléotide (NAD) : 0,75 $\mathrm{mM}$ 
- Tétra-sodium pyrophosphate (Merck) : $15 \mathrm{mM}$

- MTT Tétrazolium (Sigma) : 0,3\%o

- Phénazine méthosulphate (Sigma) : 0,02\%

2) L'autre méthode, dite "négative " (Scopes, 1968; Schapira et Nordmann, 1969) a été employée comme contrôle de la spécificité de la réaction.

Elle fait appel à la réaction inverse : l'oxaloacétate généré par le mélange acide aspartique, acide $\alpha$-cétoglutarique et transaminase glutanique oxaloacétique est réduit en acide malique avec oxydation simultanée de $\mathrm{NADH}_{2}$.

Dans un premier stade, on observe, à la lumière ultra-violette, la défluorescence des bandes correspondant à l'activité enzymatique. Ces zones sont alors visualisées en colorant le $\mathrm{NADH}_{2}$ non oxydé par une solution de gélose contenant du PMS et du MTT : elles apparaissent comme des taches blanches sur un fond bleu.

La composition du premier mélange (gel A) est la suivante :

- gélose $1 \%$

- Tampon Phosphate $\mathrm{KH}_{2} \mathrm{PO}_{4}-\mathrm{Na}_{2} \mathrm{HPO}_{4} 50$ mM, pH 7,4

- acide $\mathrm{L}(+)$ aspartique (Merck) : $20 \mathrm{mM}$

- Transaminase glutamique oxaloacétique (Boehringer) : 1 unité $/ \mathrm{ml}$.

- Nicotinamide adénine dinucléotide réduit (Sigma) : $1 \mathrm{mM}$

- Acide $\alpha$-cétoglutarique (Boehringer) : $1 \mathrm{mM}$.

La composition du second mélange (gel B) est la suivante :

- gélose $1 \%$

- MTT tétrazolium (Sigma) : $0,3 \%$

- Phénazine Methosulphate (Sigma) : 0,02\%

Pratiquement, la bande d'acétate de cellulose est disposée sur une plaque de verre couverte du gel A. Après apparition des taches de défluorescence, elle est transférée sur une seconde plaque couverte du gel $B$.

\section{NOMENCLATURE}

L'utilisation du symbole MDH étant susceptible d'introduire une confusion entre l'enzyme malate déshydrogénase et l'enzyme malique déshydrogénase (EC I.I.I.40), Shows et al. (I970) ont proposé, pour désigner la malate déshydrogénase, le symbole MOR plus conforme au nom systématique de l'enzyme recommandé par la "Commission on Enzymes of the International Union of Biochemistry ".

Dans ce système, la forme mitochondriale de la malate déshydrogénase sera Mor-I; Mor-2 étant réservé à la malate déshydrogénase cytoplasmique.

\section{RÉSULTATS}

\section{r. - Description des phénotypes}

L'enzyme étudiée présente les caractéristiques électrophorétiques de la forme mitochondriale de la malate déshydrogénase ou Mor-I. Une préparation de mitochondries purifiées suivant DE LORENZO et RUDDLE (I970) ne montre que la tache à migration anodique lente (fig. I, $a$ ).

L'électrophorèse de sang entier hémolysé ne révèle que la malate déshydrogénase cytoplasmique (isozyme Mor-2); ceci est en accord avec le fait bien connu de 1'absence de mitochondries dans le globule rouge. KARLSSON (I968), a déjà 


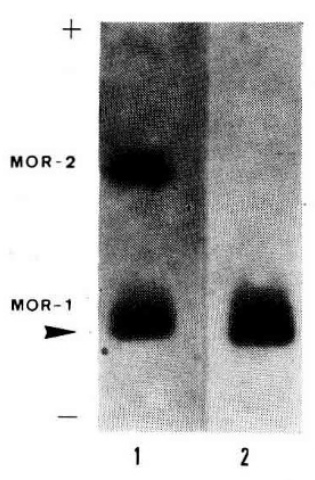

a

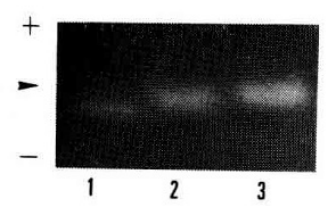

C

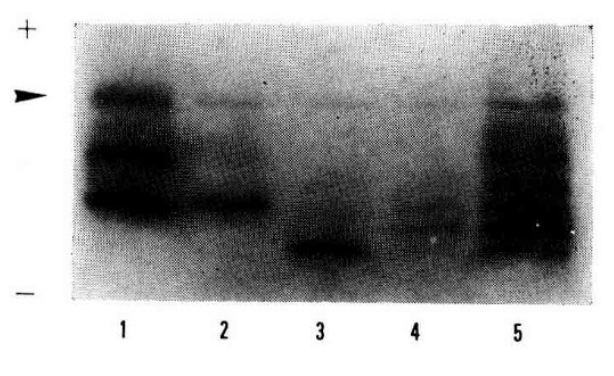

b

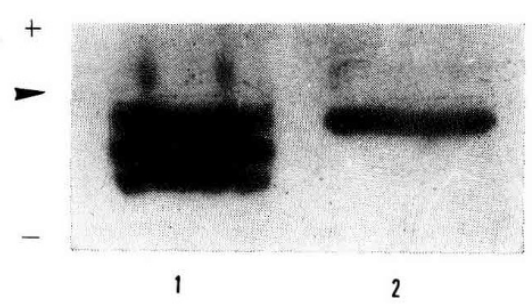

FIG. I. - a) Acétate de cellulose pH 8,6. (I) exurait musculaire total (2) extrait mitochondrial de cour de bouf. Ce zymogramme démontre la localisation mitochondriale de l'isozyme étudiè (Mor-I).

b) Gel d'amidon. Tampon de SHows et RUDDLE (1969). Une heure et demie à 500 volts.

(1) extrait inusculaire de phénotype $B B ;(2)$ leucocytes de phénotype $B B ;$; (3) leucocytes de phénotype $A A ;(4)$ lcucocytes de phinotype $A B ;(5)$ extrait musculaire de phénotype $A B$.

c) Acítate de cellulose $p H$ 8,6. Révélation négative. Globules blancs.

(I) phénotype $A A$; (2) phénotype $A B$; (3) phénotype $B B$.

d) Gel d'acrylamide. Tampon de SHOWS et RUDDLE (r969). Une heure et demie à 500 volts. Extraits musculaires.

(I) phénotype AJ'; (2) phinotype BB. Les isozymes de conformation sont absents.

montré que l'électrophorèse de sérum de porc ne révèle, pour la malate déshydrogénase, qu'une seule bande.

a) I,es zymogrammes réalisés à partir d'extraits leucocytaires, révèlent 3 phénotypes (fig. I, $b-c$ ). Deux d'entre eux sont caractérisés par une seule bande (fig. I, $b$, pistes 2 et 3 ).

Le troisième type (fig. I $b$, piste 4 ) est formé de 3 sous-bandes dont les deux extrêmes correspondent aux 2 phénotypes à une bande décrits ci-dessus; la troisième sous-bande occupe une position intermédiaire.

Ces 3 sous-bandes ne sont pas individualisées dans une électrophorèse en acétate de cellulose avec révélation négative : on n'observe alors qu'une seule bande épaisse de mobilité intermédiaire (fig. I, $c$ ).

Le mélange en proportions égales d'un extrait de chacun des types à une bande donne lieu à l'apparition de 2 bandes seulement.

b) Au départ de préparations musculaires, on obtient des images plus compliquées. Ainsi, dans le cas d'animaux répondant au phénotype leucocytaire à I bande, on obtient un ensemble de 3 sous-bandes (fig. I $b$, piste I; fig. 2). La plus lente et la plus large correspond à la bande unique des extraits leucocytaires. 
MOH MITOCHONDRIALE (MOR-I)

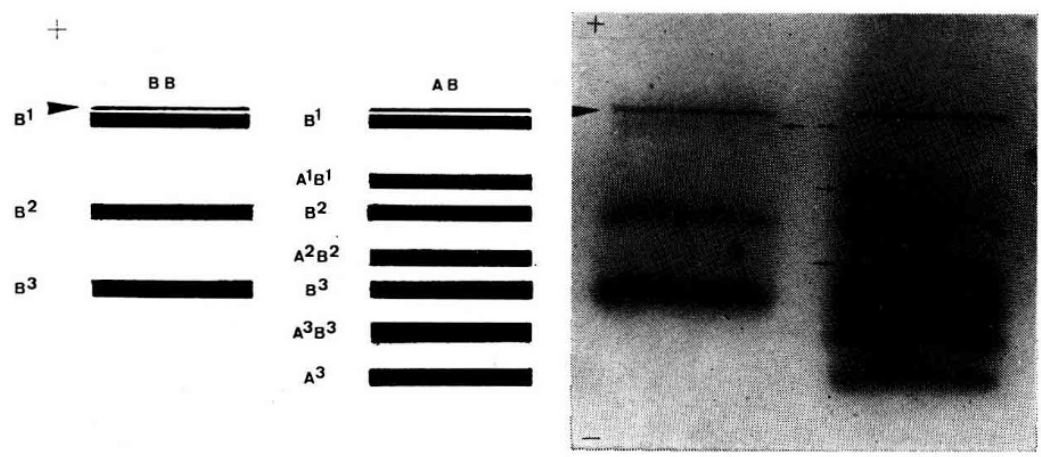

FIG. 2. - Gel d'amidon. Tampon de SHOWS et RUDDLE (I969). Une heure et demie à 500 volts. Extraits musculaires de phénotype $B B$ et de phénotype $A B$. Les sous-bandes sont identifiées à l'aide d'une nomenclature empruntée à SHOWS et al. (1970).

Par contre, chez les animaux montrant un phénotype leucocytaire à 3 bandes, on dénombre 7 sous-bandes dont les 3 plus lentes correspondent à celles du phénotype leucocytaire (fig. I $b$, piste 5 ; fig. 2).

Dans la figure 2 , ces 7 bandes ont été répertoriées et identifiées à l'aide d'une nomenclature empruntée à SHows et al. (r970).

L'électrophorèse en gel d'acrylamide paraît apporter une restriction à l'existence des sous-bandes musculaires qui n'apparaissent qu'à l'état de traces (fig. I, $d$ ).

\section{2. - Analyse génétique}

L'existence de 3 phénotypes permet de poser 1'hypothèse d'une paire d'allèles (A et $\mathrm{B}$ ) avec les 3 génctypes $\mathrm{AA}, \mathrm{BB}$ et $\mathrm{AB}$.

$\mathrm{Au}$ génotype $\mathrm{AA}$ correspondrait le phénotype leucocytaire à une bande (lente) (fig. I $b$, piste 3 ); au génotype $\mathrm{BB}$, correspondrait le phénotype à une bande (rapide) (fig. $\mathrm{I} b$, piste 2 ), tandis qu'au génotype $\mathrm{AB}$ correspondrait le phénotype à 3 bandes (fig. I $b$, piste 4 ).

Un taureau de race pie-noire, d'origine allemande, utilisé au Centre d'Insémination artificielle $\mathrm{de}$ la province de Liège avait le phénotype leucocytaire à 3 bandes correspondant au génotype $\mathrm{AB}$. Utilisé en croisement avec 24 vaches de race pie-noire, de phénotype à une bande (génotype BB), il a donné I 2 descendants de phénotype $B B$ et $I 2$ descendants de phénotype $A B$, soit les proportions attendues lors du croisement d'un hétérozygote avec des homozygotes (tabl. I).

En croisement avec 6 vaches de phénotype $A B$, on obtint 4 produits de phénotype $B B$, I de phénotype $A B$ et I de phénotype $A A$ (tab1. I). On attendait les proportions I : $2: \mathrm{I}$. On observe donc un excès de génotypes $\mathrm{BB}$ et un déficit de génotypes $A B$. Il faut toutefois tenir compte de la taille réduite de l'échantillon.

D'autre part, tous les produits d'un taureau de race de Moyenne et Haute Belgique, lui-même de phénotype à $\mathrm{I}$ bande (génotype $\mathrm{BB}$ ) et croisé avec 24 vaches 
TABLEAU I

Les phénotypes dans la descendance de différents croisements

\begin{tabular}{|c|c|c|c|c|}
\hline \multicolumn{2}{|c|}{ Phénotypes parentaux } & \multicolumn{3}{|c|}{ Phénotypes des descendants } \\
\hline$\hat{o}$ & 운 & AA & $\mathrm{AB}$ & $\mathrm{BB}$ \\
\hline $\mathrm{AB}$ & $\mathrm{BB}$ & - & 12 & 12 \\
\hline $\mathrm{AB}$ & $\mathrm{AB}$ & I & I & 4 \\
\hline BB & $\mathrm{BB}$ & - & - & 24 \\
\hline
\end{tabular}

du même phénotype, ont révélé un phénotype identique à celui de leurs parents (tabl. I).

Les résultats obtenus sont donc compatibles avec l'hypothèse faisant intervenir 2 allèles pour lesquels on peut proposer la symbolisation suivante : Mor-I-B, pour l'allèle déterminant la bande rapide et Mor-I-A pour l'allèle déterminant la bande lente.

\section{3. - Distributions raciales}

Six cents animaux, provenant pour la plupart de l'abattoir de Bruxelles ont été examinés. Les résultats sont consignés dans le tableau 2 . Le $\chi_{6}^{2}$ d'hétérogénéité est hautement significatif. La fréquence de l'allèle $A$ varie significativement d'une race à l'autre. Il est significativement plus fréquent dans la race pie noire que dans la race de Moyenne et Haute Belgique.

TABLEAU 2

La fréquence de l'allèle Mor-I-A dans quelques races

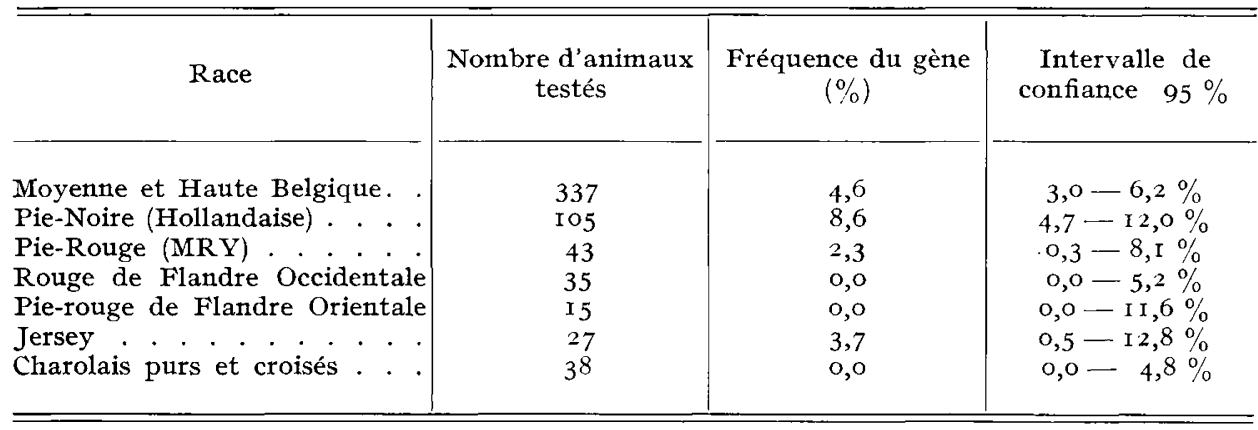




\section{DISCUSSION}

L'isozyme mitochondrial de la malate déshydrogénase, d'un poids moléculaire de 66300 , est formé de 2 sous-unités de poids moléculaire semblable ou identique (MANN et VESTLing, I969).

L'existence de nombreuses sous-bandes caractérisant les zymogrammes en gel d'amidon de la MDH mitochondriale est très bien connue depuis les travaux de Thorne et al. (I963). La nature de ces sous-bandes a été l'objet de nombreuses investigations. Suivant MANN et VesthING (I970), les 2 sous-unités moléculaires seraient différentes et se combineraient pour former 2 homodimères et I dimère hybride pour correspondre aux 3 sous-bandes habituellement observées. Beaucoup d'auteurs cependant, et cela depuis les travaux de Ki'T'o et al. (I966), KAPLAN (I968), considèrent que ces sous-bandes sont en réalité des isozymes de conformation (" conformers").

Ce terme a été proposé pour désigner les variétés isozymiques ne se distinguant que par des différences de conformation : la bande la plus cathodique, la plus importante dans le cas de la $\mathrm{MDH}$, étant l'espèce la plus compacte, la plus structurée. On a, d'autre part, souvent montré que ces isozymes pouvaient, sous certaines conditions, s'interconvertir (THORNE, I968; KITTO et al., I970).

Dans notre travail, ces isozymes de conformation apparaissent aisément en gel d'amidon ou en acétate de cellulose : ils n'apparaissent guère en gel d'acrylamide.

La présence de 3 bandes principales chez l'hétérozygote s'accorde bien avec le modèle (SHAw, I964) d'une protéine composée de 2 sous-unités : 2 types de protéines sont synthétisées correspondant respectivement aux gènes Mor-I-A et au gène Mor-I-B.

Leur association au hasard, 2 par $z$, donne les combinaisons AA, BB (homodimères), $\mathrm{AB}$ (hétérodimère). La souris, hétérozygote pour le même gène, présente également une bande intermédiaire interprétée par SHows et al. (I970) comme une molécule hétérodimère.

Les données génétiques rapportées ci-dessus sont compatibles avec la ségrégation d'une paire d'allèles codominants. Elles montrent, en même temps, que le locus correspondant au gène Mor-I est régi par le DNA nucléaire et non par le DNA mitochondrial. Cette dernière éventualité, que 1'on pouvait envisager à titre d'hypothèse, l'existence du DNA mitochondrial étant bien connue, a également été rejetée par SHows et al. (I970) à propos du même gène Mor-I de la souris.

Davidson et Cortner (I967) chez 1'homme, Longo et Scandalios (I969) chez le maïs, Shows et al. (I970) chez la souris, ont montré de semblables variants génétiques de la Mor-I.

L'enquête portant sur des animaux appartenant à des races différentes montre que la fréquence de l'allèle Mor-I-A n'est pas identique dans toutes les races, la race pie-noire (hollandaise) montrant la fréquence la plus élevée.

I,a liste des variants électrophorétiques de protéines enzymatiques s'allonge de jour en jour. Deux autres cas de cryptomorphisme sont à l'étude dans notre 
laboratoire. Ils concernent la phosphoglucomutase et la transaminase glutamique oxaloacétique.

L'emploi de globules blancs pour l'analyse des enzymes mitochondriaux, des globules rouges pour les enzymes glycolytiques notamment, devrait chez les animaux domestiques, permettre de développer ce champ d'investigations particulièrement fécond.

Rę̧u pour publication en juin 1971 .

\title{
REMERCIEMENTS
}

Nous exprimons toute notre gratitude au $\mathrm{D}^{r} \mathrm{~J}$. Marcourt, directeur du centre I.A. de la Province de Liège. Grâce à sa précieuse collaboration, nous avons pu analyser la descendance de deux taureaux I.A.

Nous remercions également F. Grosclaude et J.J. Lauvergne pour leurs suggestions concernant la présentation des résultats.

\section{SUMMARY}

\author{
H ZREDITAR ELECTROPHORETIC VARIANTS \\ OF MITOCHONDRIAI, MAIATE DEHYDROGENASE
}

Genetic varianis of the mitochondrial Malate dehydrogenase are described in the bovine species. They are revealed, by electrophoresis, in extracts from muscles as well as from leukocytes. They define a genetic system or locus, with two co-dominant alleles (suggested symbols: Mor-1-B; Mor-1-A). For the seven bre eds sampled, the frequency of the allele Mor-1-A fluctuates between 0 and 8,6 per cent.

\section{RÉFÉRENCES BIBLIOGRAPHIQUES}

Ansay, M., 1962. Microelectrophorèse en gel d'amidon. Ann. Méd. Vét., 106, r50-I54.

DAvidson, R. G., Cortner, J. A., r967. Mitochondrial malate dehydrogenase : A new genetic polymorphism in man. Science, 157, $1569-157 \mathrm{I}$.

De LoRenzo, R. J., RudDle, F. H., I970. Glutamate oxalate transaminase (GOT) genetics in Mus musculus : Linkage, polymorphism and phenotypes of the GOT-2 and GOT-x loci. Biochem. Genet., 4, $259-273$.

Florkin, M., Stotz, E. H., I964. Comprehensive Biochemistry. Volume I3. Report of the commission on enzymes of the international union of biochemistry. Elsevier Publishing Company, Amsterdam.

Hunter, R. L., MARkert, C. I.., I957. Histochemical demonstration of enzymes separated by zone electrophoresis in starch gel. Science, 125, I 294-I 295.

KAPLAN, N. O., I968. Nature of multiple molecular forms of enzymes. Ann. N. Y. Acad. Sci., 151, 382399.

KARLSSON, B. W., I968. Iactic and malic dehydrogenase activities and coenzyme patterns in blood sera of developing neonatal pigs. Biol. Neonat., 13, 34-52.

KITTo, G. B., r969. Intra- and extramitochondrial malate dehydrogenases from chicken and tuna heart. in Colowick S. P. and Kaplan N. O. Methods in Finzymology Vol. XIII Academic Press, New York, page 106.

Kitto, G. B., WASSARMax, P. M., Kaplan, N. O., I966. Enzymatically active conformers of mitochondrial malate dehydrogenase. P'roc. Natl. Acad. Sci. L.S., 56, 578-585.

KitTo, G. B., Stolzenbach, F. E., Kaplan, N. O., I970. Mitochondrial malate dehydrogenase: further studies on multiple electrophoretic forms. Biochem. Biophys. Res. Commun., 38, 3I-39.

KoHN, J. 1957. A new supporting medium for zone electrophoresis. Biochem. J., 65, 90.

I,ongo, G. P., Scandalios, J. G., I969. Nuclear gene control of mitochondrial malic dehydrogenase in maize. Proc. Natl Acad. Sci., 62 : 104. 
Mann, K. G., Vestling, C. S. I969. Subunits of rat liver mitochondrial malate dehydrogenase. Biochemistry, 8, I I05-I rog.

Mann, K. G., Vestling, C. S., I97o. Isozymes of rat liver mitochondrial malate dehydrogenase. Evidence for the existence of nonidentical subunits. Biochemistry, 9, 3020-3025.

MARKert, C. L., WhitT, G. S., I968. Molecular varieties of isozymes. Experientia, 24, 977-99 I.

Schaptra, F., NordmanN, Y., r969. Présence de trois types d'aldolase dans le foie humain. Clin. Chim. Acta, 26, I89-195.

Scopes, R. K., I 968 . Methods for starch-gel electrophoresis of sarcoplasmic proteins. An investigation of the relative mobilities of the glycolytic enzyme from the muscles of a variety of species. Biochem. J., 107, 139-ז 50.

SHAw, C. R., I964. The use of genetic variation in the analysis of isozyme structure. Brookhaven Symp. Biol., 17, I I7-I29.

Shows, T. B., Rudder, F. H., RoDerick, T. H. I969. Phosphoglucomutase electrophoretic variants in the mouse. Biochem. Genet., 3, 25-33.

Shows, T. P., Chapman, V. M., Ruddle, F. H., I970. Mitochondrial malate dehydrogenase and malic. enzyme : mendelian inherited electrophoretic variants in the mouse. Biochem. Genet., 4, 707-718.

Smrthies, O., 1955. Zone electrophoresis in starch-gels : group variations in the serum proteins of normal human adults. Biochem. J., 61, 629-64I.

Smrthres, O., I959. An improved procedure for starch-gel electrophoresis: further variations in the serum proteins of normal individuals. Biochem. J., 71, 585-587.

Thorne, C. J. R., I960. Characterisation of two malic dehydrogenase from rat liver. Biochem. Biophys. Acta, 42, I75. I76.

ThorNe, C.J.R., I968. Interconversion of the components of rat brain mitochondrial malate dehydrogenase. FEBS, Letters, 1, 24I-243.

Thorne, C.J.R., Grossman, I.I., Kaplan, N.O., I963. Starch-gel electrophoresis of malate dehydrogenase. Biochim. Biophys. Acta, 73, I93-203.

Uriel, J., I966. Méthode d'électrophorèse dans des gels d'acrylamide-agarose. Bull. Soc. Chim. Biol., $48,969-982$.

Wieme, R. J., 1965. Agar gel electrophoresis. Elsevier Publishing Company, Amsterdam. 